\title{
Floristic Diversity of the Sacred Grove of Madathody Naga Kavu, Chalavara, Palakkad District, Kerala State
}

\author{
Praveenkumar K* \\ P.G and Research Department of Botany, Sree Krishna College, Guruvayur, Ariyannur (P.O), Thris sur District, Kerala 680102 \\ praveenkumarkvk1992@gmail.com
}

\begin{abstract}
An exploratory survey conducted in Madathody Nagakavu is present in Chalavara Gramapanchayath, Ottapalam taluk of Palakkad district, Kerala lead to the collection of 50 species coming under 49 genera and 25 families. Among them, 6 endemic, rare, and red listed plants are represented here and also including 38 medicinal plants.
\end{abstract}

Keywords-Diversity, Madathody Naga kavu, Palakkad.

\section{INTRODUCTION}

Sacred groves are the conserved forest patches, or protected areas. The sacred groves in Kerala are tightly bound with religional backgrounds. The conservation is mainly based on cultural, aesthetical, and religion aspects, and in different areas these sacred groves are devoted or dedicated for different Gods ie. serpentine Gods, Nagadevatha, Nagayakshi etc. Sacred groves are one of the informal approaches of conserving the biological diversity of a region and play an important role in preservation of depleting resource elements such as medicinal plants and occur in India and in other parts of Asia and Africa (Bhandary and Chandrashekar, 2003). According to Nair (1992) in sacred groves the number of herbs and shrubs are reported to be more in the disturbed zone. Due to urbanization and industrialization coupled with rationalization, scarcity of land leading to the depletion of the cover and shrinkage of these areas as a result the large chunk of the areas are diverted for other activities and only a small portion is left with especially adjacent to the temple (Devaraj., et al 2005). According to Rajasri Ray et al., (2014) sacred groves may lose their prominence nowadays, but are still relevant in Indian rural landscapes inhabited by traditional communities.

A rough estimate Kerala has about 1500 sacred groves, which are distinct and biologically unique. Sacred groves in Kerala are located mainly in Kasargod, Kannur,
Kozhikode, Thrissur, Palakkad, Ernakulam and Alappuzha districts. Balasubramanyan and Induchoodan (1999) recorded a total number of 761 sacred groves in Kerala State.

In Kerala the common practice among Hindus to assign a part of their land near the Tharavadu or house as the abode of goddess Durga or serpent god Naga or Shasta and the place is called 'Kavu' or 'Sarpakavu'. People are prohibited from felling trees and even removing a twig is considered as taboo. Apart from conserving biological diversity, sacred groves that are situated in the middle of the human habitation are responsible for conserving water and soil.

\section{STUDY AREA}

Madathody Naga Kavu is one among this and located near Shornur, Chalavara Grama Panchayath of Palakkad district. The management of this kavu is under the control of Madhathody family. The area is about 36 cent. The deity is Nagam. A mud road splits this kavu in to two parts. The present study conducted in the Madathody NagaKavu has resulted in the collection of 50 taxa of angiosperms coming under 49 genera and 25 families. Out of these, 06 rare, endemic, red listed and taxonomically important species are enumerated here. (Table 1). The voucher specimens are deposited at the Sree Krishna College, Guruvayur.

\section{MATERIALS AND METHODS}

The Sacred grove was visited during different seasons and two specimens were collected in each species and these were systematically numbered and tagged. Important field observation like, habit, phenology of the plant, colour, texture and smell of leaves, abundance, local names and local uses available were also noted. Each species in fresh condition was critically studied with the 
help of floras like, Flora of Presidency of Madras (Gamble, 1915-1936); . The plants were identified with the help of floras and finally by comparing with the reference collections available in the Herbarium of Kerala Forest Research Institute, Peechi. The species were often pois oned, processed and labeled, by standard herbarium methods given by Santapau (1955) and Jain \& Rao (1977).

\section{RESULTS AND DISCUSSION}

The present study conducted in the Madathody NagaKavu has resulted in the collection of 50 taxa of angiosperms coming under 49 genera and 25 families including 19 herbs, 13 shrubs 10 trees and 8 climbers. Out of 50 species 6 plants are Endemic, 8 Exotic and 38 medicinally important were collected. (Table 1).

Table.1: Species recorded from Madathody kavu

\begin{tabular}{|c|c|c|c|c|c|c|c|}
\hline $\begin{array}{l}\text { Sl. } \\
\text { No }\end{array}$ & $\begin{array}{l}\text { Coll. } \\
\text { No. }\end{array}$ & Botanical name & Family & Local name & Habit & $\begin{array}{l}\text { System of } \\
\text { Medicine }\end{array}$ & Status \\
\hline & 105 & $\begin{array}{l}\text { Ichnocarpus frutescens } \\
\text { (L.) R.Br. }\end{array}$ & Apocynaceae & & Climber & $\mathrm{A}, \mathrm{F}, \mathrm{S}, \mathrm{T}, \mathrm{U}$ & \\
\hline & 196 & $\begin{array}{l}\text { Mikania micrantha Kunth } \\
\text { in HBK }\end{array}$ & Asteraceae & & Climber & - & \\
\hline & 190 & $\begin{array}{l}\text { Merremia vitifolia } \\
\text { (Burm.f.) Hall.f. }\end{array}$ & Convolvulaceae & & Climber & $\mathrm{F}$ & \\
\hline & 118 & $\begin{array}{l}\text { Luffa cylindrica (L.) } \\
\text { M.J.Roem }\end{array}$ & Cucurbitaceae & & Climber & $\mathrm{A}, \mathrm{S}, \mathrm{T}$ & \\
\hline & 129 & Dioscorea bulbifera L. & Dioscoreaceae & & Climber & $\mathrm{A}, \mathrm{F}, \mathrm{S}, \mathrm{T}, \mathrm{U}$ & \\
\hline & 131 & $\begin{array}{l}\text { Derris scandens (Roxb.) } \\
\text { Benth. }\end{array}$ & Fabaceae & & Climber & - & \\
\hline & 207 & $\begin{array}{l}\text { Asparagus racemosus, } \\
\text { Willd. }\end{array}$ & Liliaceae & & Climber & $\mathrm{A}, \mathrm{F}, \mathrm{S}, \mathrm{T}, \mathrm{U}$ & \\
\hline & 214 & Glorios a superba L. & Liliaceae & Menthonny & Climber & $\mathrm{A}, \mathrm{F}, \mathrm{S}, \mathrm{T}, \mathrm{U}$ & \\
\hline & 100 & $\begin{array}{l}\text { Andrographis paniculata } \\
\text { (Burm.f.) Wall.ex.Nees. }\end{array}$ & Acanthaceae & Gopuramthangi & Herb & $\mathrm{A}, \mathrm{F}, \mathrm{H}, \mathrm{S}, \mathrm{U}$ & \\
\hline & 205 & Ageratum conyzoidsL. & Asteraceae & & Herb & $\mathrm{A}, \mathrm{F}, \mathrm{S}$ & \\
\hline & 215 & $\begin{array}{l}\text { Emilia sonchifolia (L.) } \\
\text { DC. in Wight }\end{array}$ & Asteraceae & & Herb & $\mathrm{A}, \mathrm{F}, \mathrm{S}, \mathrm{U}$ & \\
\hline & 102 & Tridax procumbens L. & Asteraceae & & Herb & $\mathrm{F}, \mathrm{S}$ & Exotic \\
\hline & 204 & $\begin{array}{l}\text { Cleome burmannii Wight } \\
\text { \&Arn. }\end{array}$ & Capparidaceae & & Herb & - & \\
\hline & 181 & $\begin{array}{l}\text { Euphorbia thymifolia } \\
\text { Linn. }\end{array}$ & Euphorbiaceae & & Herb & $\mathrm{A}, \mathrm{F}, \mathrm{U}$ & \\
\hline & 165 & $\begin{array}{l}\text { Phyllanthus urinaria, } \\
\text { Linn. }\end{array}$ & Euphorbiaceae & & Herb & $\mathrm{A}, \mathrm{S}, \mathrm{U}$ & \\
\hline & 196 & $\begin{array}{l}\text { Sebastiana chamaelea (L.) } \\
\text { Muell.-Arg. }\end{array}$ & Euphorbiaceae & & Herb & $\mathrm{F}$ & \\
\hline & 103 & $\begin{array}{l}\text { Leucas aspera (Willd.) } \\
\text { Spreng. }\end{array}$ & Lamiaceae & Thumba & Herb & $\mathrm{A}, \mathrm{F}, \mathrm{H}, \mathrm{S}, \mathrm{U}$ & \\
\hline & 176 & Osbeckia muralis Naud. & Melastomataceae & & Herb & - & Endemic \\
\hline & 108 & Mimosa pudica L. & Mimosaceae & & Herb & $\mathrm{A}, \mathrm{F}, \mathrm{S}, \mathrm{T}, \mathrm{U}$ & Exotic \\
\hline & 201 & Boerhaavia diffusa Linn. & Nyctaginaceae & Thazhuthama & Herb & $\mathrm{A}, \mathrm{F}, \mathrm{H}, \mathrm{S} \mathrm{U}$ & \\
\hline & 147 & $\begin{array}{l}\text { Desmodium gangeticum } \\
\text { (L.) DC. }\end{array}$ & Fabaceae & & Herb & - & \\
\hline & 210 & $\begin{array}{l}\text { Desmodium scorpiurus } \\
\text { (Sw.) Desv. }\end{array}$ & Fabaceae & & Herb & - & \\
\hline
\end{tabular}




\begin{tabular}{|c|c|c|c|c|c|c|}
\hline 122 & Panicum notatum Retz. & Poaceae & & Herb & - & \\
\hline 128 & $\begin{array}{l}\text { Sporobolus diander } \\
\text { (Retz.) P. Beauv. }\end{array}$ & Poaceae & & Herb & - & \\
\hline 169 & Oldenlandia umbellata L. & Rubiaceae & & Herb & - & \\
\hline 181 & Spermacoce latifolia Aubl & Rubiaceae & & Herb & - & Exotic \\
\hline 133 & $\begin{array}{l}\text { Lindernia ciliata (Colsm.) } \\
\text { Pennell. }\end{array}$ & Scrophulariaceae & & Herb & - & \\
\hline 178 & Eupatorium odoratum L. & Asteraceae & $\begin{array}{l}\text { Communist } \\
\text { pacha }\end{array}$ & Shrub & $\mathrm{F}$ & Exotic \\
\hline 203 & $\begin{array}{l}\text { Briedelia scandens } \\
\text { (Roxb.) Willd. }\end{array}$ & Euphorbiaceae & & Shrub & $\mathrm{F}$ & Endemic \\
\hline 109 & $\begin{array}{l}\text { Sauropus androgynous } \\
\text { (L.) Merr. }\end{array}$ & Euphorbiaceae & & Shrub & $\mathrm{F}, \mathrm{S}$ & \\
\hline 265 & $\begin{array}{l}\text { Hyptis suaveolens (L.) } \\
\text { Poit. }\end{array}$ & Lamiaceae & & Shrub & $\mathrm{F}$ & Exotic \\
\hline 134 & $\begin{array}{l}\text { Leea indica (Burm.f.) } \\
\text { Merr. }\end{array}$ & Leeaceae & & Shrub & $\mathrm{A}, \mathrm{F}, \mathrm{S}$ & \\
\hline 149 & $\begin{array}{l}\text { Hibiscus his pidis simus } \\
\text { Griff. }\end{array}$ & Malvaceae & & Shrub & $\mathrm{A}$ & \\
\hline 171 & $\begin{array}{l}\text { Sida rhomboidea Roxb. } \\
\text { ex Fleming. }\end{array}$ & Malvaceae & & Shrub & AFSTU & Endemic \\
\hline 186 & $\begin{array}{l}\text { Memecylon randerianum } \\
\text { SM \& MR Almeida }\end{array}$ & Melastomataceae & & Shrub & $\mathrm{F}$ & Endemic \\
\hline 153 & $\begin{array}{l}\text { Chassalia curviflora (Wall } \\
\text { ex Kurz) Thw. }\end{array}$ & Rubiaceae & & Shrub & $\mathrm{F}$ & \\
\hline 101 & Ixora coccinea $\mathrm{L}$. & Rubiaceae & & Shrub & $\mathrm{A}, \mathrm{F}, \mathrm{S} \mathrm{U}$ & \\
\hline 163 & $\begin{array}{l}\text { Grewia nervosa(Lour.) } \\
\text { Panigrahi }\end{array}$ & Tiliaceae & & Shrub & $\mathrm{F}$ & \\
\hline 166 & $\begin{array}{l}\text { Triumfetta rhomboidea } \\
\text { Jacq. }\end{array}$ & Tiliaceae & & Shrub & $\mathrm{F}, \mathrm{S}, \mathrm{U}$ & \\
\hline 211 & $\begin{array}{l}\text { Clerodendrum } \\
\text { infortunatum L. }\end{array}$ & Verbenaceae & & Shrub & $\mathrm{A}, \mathrm{F}$ & \\
\hline 219 & Mangifera indica L. & Anacardiaceae & Mavu & Tree & $\mathrm{A}, \mathrm{F}, \mathrm{H}, \mathrm{T}, \mathrm{U}$ & \\
\hline 153 & Plumeria rubra L. & Apocyanaceae & & Tree & $\mathrm{A}, \mathrm{F}$ & Exotic \\
\hline 104 & $\begin{array}{l}\text { Alstonia scholaris (L.) R. } \\
\text { Br. }\end{array}$ & Apocynaceae & & Tree & $\mathrm{A}, \mathrm{F}, \mathrm{H}, \mathrm{T}, \mathrm{U}$ & \\
\hline 213 & Caryota urens Linn. & Arecaceae & & Tree & $\mathrm{A}, \mathrm{F}, \mathrm{S}, \mathrm{U}$ & \\
\hline 125 & Cocos nucifera L. & Arecaceae & Thengu & Tree & $\mathrm{A}, \mathrm{F}, \mathrm{S}, \mathrm{T}, \mathrm{U}$ & \\
\hline 106 & Acacia mangium Willd. & Mimosaceae & & Tree & - & Exotic \\
\hline 174 & Artocarpus hirsutus Lam. & Moraceae & & Tree & $\mathrm{A}, \mathrm{F}$ & Endemic \\
\hline 209 & Ficus racemosa L. & Moraceae & & Tree & $\mathrm{A}, \mathrm{F}, \mathrm{S}, \mathrm{T}, \mathrm{U}$ & \\
\hline 218 & Olea dioica Roxb. & Oleaceae & & Tree & $\mathrm{F}, \mathrm{S}$ & Endemic \\
\hline 169 & $\begin{array}{l}\text { Gliricidia sepium (Jacq.) } \\
\text { Kunth ex Walp. }\end{array}$ & Fabaceae & & Tree & $\mathrm{F}$ & Exotic \\
\hline
\end{tabular}

A: Ayurveda, F: Folk, S: Siddha, U: Unani, H: Homoeopathy, T: Tibetan, M: Modern. 


\section{CONCLUSION}

Sacred groves are considered as store house of rare, endemic and endangered plants because of floristic wealth and biodiversity conservation. Some sacred groves are still remaining in undisturbed state and they help to conserve biodiversity and ecological balance. Medicinal and economically important plants are also present in sacred groves. Due to several construction works in sacred groves, a wide range of flora is disturbed; their extensive population in nature is gradually diminishing.The total area of this kavu is unprotected due to this, exotic weeds are invading to this area. Here endemic plants Artocarpus hirsuta, Bridelia scandens, Memecylon malabaricum, Osbeckia muralis, and Olea dioica are present. Out of 50 plants 38 are medicinal. Large number of herbs and shrubs are present, but numbers of trees are very less. This shows the disturbance inside the grove. In this circumstance suitable management measures and awareness programmes about the importance of sacred groves are necessary for sustainable utilization of the valuable bioresources Chalavara Grama panchayath proposed suitable plans to protect these sacred groves.

\section{ACKNOWLEDGEMENTS}

I am much grateful to Dr. G. Jayakrishnan, Department of Botany, Sree Krishna College, Guruvayur for providing valuable suggestions for the work. We are thankful to the members of Madhathody family for granting permission to conduct the study and providing information about the sacred grove.

\section{REFERENCES}

[1] Balasubramanyan, K. and Induchoodan, N.C. 1999. Can the endemics of the Sacred Groves in Kerala withstand human onslaught? In: G. Kumaravelu and K.K. Chaudhuri (eds) Endemic and endangered plant and animal species of Eastern and Western Ghats. Proceedings of the national seminar conducted by Research Wing, Tamil Nadu Forest Department, at Chennai, pp.59-64.

[2] Bhandary, M.J. and Chandrashekar, K.R. 2003. Sacred Groves of Dakshina Kannada and Udupi districts of Karnataka. Curr. Sci. 85(12): 1655-1656.

[3] Devaraj, P., Ramanujam M.P., and Ganesan, T. 2005 Status report of Sacred groves of Pondicherry Region and Strategies for Conservation, Institute of Forest Genetics and Tree Breeding PB 1061, R.S. Puram, Coimbatore 641002, India. pp. 16-21.
[4] Gamble, J.S. and C.E.C. Fischer, 1915-1936. The Flora of the Presidency of Madras. parts 1-11 (parts 17 by Gamble and $8-11$ by Fischer), Vols. 1-3. Adlard\& Sons Ltd., London.

[5] Jain, S.K. and R.R. Rao, 1977. A Handbook of Field and Herbarium Methods. Today \& Tomorrow, New Delhi.

[6] Nair, H.G. 1992. Ecological studies of a Sacred Grove. Project report submitted to W.W.F, New Delhi. pp.55.

[7] Rajasri Ray; Chandran, M. D. S. and Ramachandra, T. V. 2014. Biodiversity and ecological assessments of Indian sacred groves. Northeast Forestry University, Harbin, China, Journal of Forestry Research. 25 (1). pp. 21-28.

[8] Santapau, H. 1955. Botanical Collector's manual - A Handbook. New Delhi. 